\title{
A retrospective study of the impact of health worker strikes on maternal and child health care utilization in western Kenya
}

Michael L. Scanlon ${ }^{1,2^{*}}$, Lauren Y. Maldonado ${ }^{2,3}$, Justus E. Ikemeri², Anjellah Jumah², Getrude Anusu², Jeffrey N. Bone ${ }^{4}$, Sheilah Chelagat ${ }^{2}$, Joann Chebet Keter ${ }^{2}$, Laura Ruh2 ${ }^{2,5}$, Julia Songok ${ }^{2,6}$ and Astrid Christoffersen-Deb $2,4,7$

\begin{abstract}
Background: There have been dozens of strikes by health workers in Kenya in the past decade, but there are few studies of their impact on maternal and child health services and outcomes. We conducted a retrospective survey study to assess the impact of nationwide strikes by health workers in 2017 on utilization of maternal and child health services in western Kenya.

Methods: We utilized a parent study to enroll women who were pregnant in 2017 when there were prolonged strikes by health workers ("strike group") and women who were pregnant in 2018 when there were no major strikes ("control group"). Trained research assistants administered a close-ended survey to retrospectively collect demographic and pregnancy-related health utilization and outcomes data. Data were collected between March and July 2019. The primary outcomes of interest were antenatal care (ANC) visits, delivery location, and early child immunizations. Generalized estimating equations were used to estimate risk ratios between the strike and control groups, adjusting for socioeconomic status, health insurance status, and clustering. Adjusted risk ratios (ARR) were calculated with $95 \%$ confidence intervals (95\%Cl).

Results: Of 1341 women recruited in the parent study in 2017 (strike group), we re-consented 843 women (63\%) to participate. Of 924 women recruited in the control arm of the parent study in 2018 (control group), we reconsented 728 women (79\%). Women in the strike group were $17 \%$ less likely to attend at least four ANC visits during their pregnancy (ARR $0.83,95 \% \mathrm{Cl} 0.74,0.94)$ and $16 \%$ less likely to deliver in a health facility (ARR $0.84,95 \% \mathrm{Cl}$ $0.76,0.92)$ compared to women in the control group. Whether a child received their first oral polio vaccine did not differ significantly between groups, but children of women in the strike group received their vaccine significantly longer after birth ( 13 days versus 7 days, $p=0.002$ ).

Conclusion: We found that women who were pregnant during nationwide strikes by health workers in 2017 were less likely to receive WHO-recommended maternal child health services. Strategies to maintain these services during strikes are urgently needed.
\end{abstract}

Keywords: Health worker strike, Maternal child health, Kenya

\footnotetext{
* Correspondence: mscanlon@iu.edu

IIndiana University Center for Global Health, 702 Rotary Circle, Suite RO 101, Indianapolis, Indiana, USA

${ }^{2}$ Academic Model Providing Access to Healthcare (AMPATH), Eldoret, Kenya

Full list of author information is available at the end of the article
} 


\section{Background}

Strikes by health workers are not new phenomena but there is evidence that they may be increasing globally and represent an important but under-explored challenge to initiatives for universal health coverage [1]. In the past decade, health workers in Kenya have launched major strikes in the public health sector to protest a wide range of issues, including low pay, poor working conditions, and implementation of collective bargaining agreements, among others [2]. In 2017, public sector physicians, nurses, and clinical officers were on separate nationwide strikes for a combined 250 days. Physicians were on strike for 100 days (December 5, 2016 to March 15,2017 ) and nurses were on strike for 150 days (June 5 , 2017 to November 2, 2017). Clinical officers, who are mid-level, non-physician clinicians in Kenya [3], launched their own 20-day strike in the midst of the nurses' strikes. Strikes in Kenya are associated with significant decreases in inpatient and outpatient admissions in public facilities $[4,5]$, but their association with health outcomes including morbidity and mortality is less clear [6-8]. The impact of strikes on essential maternal and child health services and outcomes has not been adequately explored [9].

Maternal and neonatal mortality is a major public health issue in Kenya [10, 11]. In 2017, the maternal mortality ratio was estimated to be 342 per 100,000 live births [12], and data from the most recent Demographic and Health Survey in 2014 found that only about $60 \%$ of women attended four or more antenatal care (ANC) visits and delivered with a skilled birth attendant [13]. Recent government reforms have aimed to improve access and utilization of maternity services [14]. In 2017, the government launched a new scheme called Linda Mama as part of its strategy to progress towards universal health coverage. The program is eligible to all Kenyan citizens and covers maternity services including ANC, delivery, and postnatal care under the national health insurer, the National Hospital Insurance Fund, at accredited public and private health facilities [15]. Data show that these policies have likely contributed to increases in ANC and delivery with a skilled birth attendant, but implementation of these policies has been inconsistent due to significant demand- and supply-side barriers, including systemic inequities particularly for women who are poorer and reside in more rural communities [16-20]. It is unclear how recent strikes have affected the implementation of these policies and potentially exacerbated inequities in service coverage and utilization.

To investigate the impact of the prolonged strikes by health workers in Kenya on maternal and child health care utilization, we conducted a natural experiment using retrospective surveys with women who were pregnant in 2017 during health worker strikes and women who were pregnant in 2018 when there were no major strikes.

\section{Methods \\ Study setting}

This study was conducted in Trans Nzoia County in western Kenya. Trans Nzoia has a population of approximately one million people with a largely rural and agricultural economy, and generally scores below national averages on primary and maternal child health indicators such as ANC, facility deliveries, and child immunizations [21]. In the most recent Kenya Demographic and Health Survey, the percentage of women who delivered in a health facility was $41.5 \%$ in Trans Nzoia compared to $61.2 \%$ nationally [13]. According to the Kenya Ministry of Health Master Health Facility List, Trans Nzoia has 182 registered health facilities, which includes seven public hospitals, five private hospitals, one faith-based hospital, and one County referral hospital [22]. In addition to national-level strikes by health workers, Trans Nzoia nurses employed in public sector facilities launched their own 44-day strike across the County from February 1, 2017 to March 23, 2017 to protest delays in salaries, remittance of statutory deductions, and promotions [23]. This meant that in Trans Nzoia either physicians, nurses, or clinical officers were on strike in the public health sector for a total of 232 days in 2017. We are not aware of any major strikes by health workers in private or faith-based facilities during the study period.

\section{Study design}

We utilized a parent study to conduct a natural experiment and retrospectively assessed the impact of the 2017 strikes on the utilization of maternal and child health services. As part of the parent study called Chamas for Change, we recruited a cohort of pregnant women in 2017 and a different cohort of pregnant women in 2018 when there were no major strikes by health workers [24]. In 2019, we traced women previously recruited in the parent study, re-consented them in the current study, and administered a close-ended questionnaire. We also conducted focus group discussions with a subset of these women and community health volunteers (CHVs) as well as in-depth interviews with health facility managers, which is reported elsewhere [25].

The parent study, Chamas for Change, was a cluster randomized controlled trial to evaluate the effectiveness of a CHV-led, group-based model of care to improve maternal and child health in Trans Nzoia County in western Kenya. In 2017, pregnant women who presented for ANC services at one of 74 public or private health 
facilities in Trans Nzoia were recruited into the parent study. All participants provided informed consent to participate in the parent study and completed a baseline assessment. Prior to launching the intervention, nurses launched their nationwide strike, and the parent study was halted. In 2018, a new cohort of pregnant women was recruited to participate in the trial using the same enrollment criteria. Additional details on the parent study are provided elsewhere [24].

\section{Participants}

Participants were women who were recruited in the Chamas for Change parent study in 2017 ("strike group") and in the control arm of the trial in 2018 ("control group"). Women in the strike group and the control group did not receive any intervention as part of the parent study and met the same inclusion criteria at the time of enrollment into the parent study: 18 years of age or older, pregnant, and less than 24 weeks gestation based on last menstrual period. Using contact information provided as part of the parent study, we contacted women to see if they would be interested in participating in this study. Participants completed an interview using a close-ended questionnaire about their pregnancy and pregnancy-related health utilization and outcomes with a trained research assistant.

\section{Data collection}

Data were collected between March and July 2019. All participants provided informed consent to participate. Interviews using close-ended questionnaires were conducted in English or Kiswahili by trained research assistants at participants' homes or by phone if they had moved outside of Trans Nzoia. All data were collected via computer tablets using a mobile REDCap survey application and synchronized to a central REDCap database twice weekly $[26,27]$. The interview took approximately $30 \mathrm{~min}$ to complete. Demographic and socioeconomic characteristics were collected in addition to data on pregnancy-related services and outcomes. For in-person interviews, women were asked to share their Mother-Baby Booklet that is provided by the Kenya Ministry of Health that contains pregnancy and delivery information. In cases where the Mother-Baby Booklet was not available and for participants who were contacted by phone, women were asked to self-report.

\section{Analysis}

Data were analyzed descriptively for demographic, socioeconomic, and pregnancy-related outcomes. We compared demographics and socio-economic status between women in the strike versus control group using Student's t-tests for normally distributed continuous variables and Pearson's chi-squared tests for categorical variables. Generalized estimating equations (to account for clustering) with a Poisson link were used to estimate the risk ratios between the strike and control group for outcomes of interest [28]. As in the parent study [24], the primary outcomes of interest were ANC visits, facility-based delivery, and early child immunizations. There were high rates of missing immunization data (>50\%) other than the first oral polio vaccine, and so additional immunizations were excluded in the analysis. Models were adjusted for socioeconomic status and insurance status at delivery. For continuous outcomes, a Gaussian link rather than a Poisson was used. Adjusted risk ratios (ARR) are presented with 95\% confidence intervals $(95 \% \mathrm{CI})$.

\section{Results}

\section{Participant characteristics}

Of 1341 women recruited in the parent study in 2017 (strike group), we successfully located and re-consented 843 women $(63 \%)$ to participate in close-ended interviews using the contact information provided during their initial enrollment. Of 924 women recruited in the control arm of the parent study in 2018 (control group), 728 women $(79 \%)$ were located and re-consented. The average number of days between delivery and the study interview was 611 days (standard deviation, SD, 63 days) for women in the strike group and 324 days (SD 68 days) for women in the control group. Most interviews were conducted in-person by research assistants $(84 \%$ in the strike group versus $88 \%$ in the control group) with the remainder conducted by phone.

Demographic characteristics of participants who completed close-ended interviews are presented in Table 1. There were no meaningful differences in age, marital status, employment status, or poverty level between women in the strike versus control group. Women in the control group were significantly more likely to have health insurance at the time of delivery compared to women in the strike group (59\% versus 15\%). The majority of women in the strike group $(76 \%)$ reported being aware of a health worker strike during their pregnancy. In the control group, $4 \%$ of women also reported being aware of a health worker strike during their pregnancy even though we could not find any report of strikes in this period. Most women (78\%) in the strike group gave birth during the nurses' strike from June 5 to November 2 2 2017, with the remainder either having a missing delivery date or reporting a delivery date after the nurses' strike ended.

Maternal and child health care utilization and outcomes Women in the strike group were $17 \%$ less likely to attend at least four ANC visits during their pregnancy (ARR $0.83,95 \%$ CI $0.74,0.94$ ) and $16 \%$ less likely to 
Table 1 Characteristics of participants who completed close-ended interview

\begin{tabular}{lllc}
\hline Characteristic & $\begin{array}{l}\text { Strike group (women pregnant in 2017) } \\
\boldsymbol{n = 8 4 3}\end{array}$ & $\begin{array}{l}\text { Control group (women pregnant in 2018) } \\
\boldsymbol{n = 7 2 8}\end{array}$ & $\boldsymbol{p}$-value \\
\hline \% reconsented from parent study & $63 \%$ & $79 \%$ & $\mathrm{n} / \mathrm{a}$ \\
Mean age, years (SD) & $27.0(5.9)$ & $27.1(6.2)$ & 0.83 \\
Marital status, married & $684(81 \%)$ & $606(83 \%)$ & $272(37 \%)$ \\
Employed (formally or informally) & $349(41 \%)$ & $48.8(20.1)$ & 0.74 \\
Mean poverty index score (SD) ${ }^{a}$ & $50.6(20.0)$ & $412(59 \%)$ & 0.10 \\
Health insurance at delivery & $120(15 \%)$ & 0.07 \\
\hline
\end{tabular}

${ }^{a}$ We used the 10-item Poverty Probability Index for Kenya (https://www.povertyindex.org/country/kenya) created by Innovations for Poverty Action (version October 2018) that is based on Kenya's 2015 Integrated Household Budget Survey

deliver in a health facility (ARR $0.84,95 \%$ CI $0.76,0.92$ ) compared to women in the control group (Table 2). Among women who delivered in a health facility, women in the strike group were more likely to deliver in a private facility (38\%) compared to women in the control group (11\%). Only $33 \%$ of women in the strike group reported that they delivered their child at their preferred location compared to $73 \%$ of women in the control group. There were no differences in livebirth or first oral polio vaccine (OPV 0) rates. Data for complications during delivery and $\mathrm{CHV}$ visit within 48 hours of birth were inconclusive. Children of women in the strike group received their first OPV 0 vaccine significantly later after birth compared to the control group (13 days versus seven days, mean difference $=8.02,95 \% \mathrm{CI}=3.06,12.98$ ) .

\section{Discussion}

We found that recent strikes by health workers were associated with lower maternal and health care utilization of ANC and delivery at a health facility among pregnant women in Trans Nzoia County in western Kenya. We also found evidence that strikes were associated with

Table 2 Maternal and child health outcomes

\begin{tabular}{|c|c|c|c|}
\hline Outcome & $\begin{array}{l}\text { Strike group } \\
\text { (women pregnant in 2017) } \\
n=843\end{array}$ & $\begin{array}{l}\text { Control group } \\
\text { (women pregnant in 2018) } \\
n=728\end{array}$ & $\begin{array}{l}\text { Adjusted risk ratio } \\
{[95 \% \mathrm{Cl}] ; \boldsymbol{p} \text {-value }}\end{array}$ \\
\hline 4 or more ANC visits, $\mathrm{n}(\%)$ & $463(55 \%)$ & $507(70 \%)$ & $0.83[0.74,0.94] ; 0.004$ \\
\hline Delivered in a facility (public or private), $\mathrm{n}(\%)$ & $452(55 \%)$ & $514(73 \%)$ & $0.84[0.76,0.92] ;<0.001$ \\
\hline Delivery location, n (\%) & & & $\mathrm{n} / \mathrm{a}$ \\
\hline Home alone & $75(9 \%)$ & $43(6 \%)$ & \\
\hline Home with TBA & $284(34 \%)$ & $139(20 \%)$ & \\
\hline In transit & $14(2 \%)$ & $8(1 \%)$ & \\
\hline Private facility & $316(38 \%)$ & $77(11 \%)$ & \\
\hline Public facility & $136(17 \%)$ & $437(62 \%)$ & \\
\hline Delivered at preferred location, n (\%) & $268(33 \%)$ & $514(73 \%)$ & $0.49[0.42,0.58] ;<0.001$ \\
\hline Any delivery complications, $n(\%){ }^{a, b}$ & $113(19 \%)$ & $132(25 \%)$ & $0.81[0.59,1.11] ; 0.185$ \\
\hline Delivered a live child, n (\%) & 794 (94\%) & $696(96 \%)$ & $1.00[0.97,1.02] ; 0.711$ \\
\hline Mean child birthweight, kgs (SD) & $3.40(0.66)$ & $3.43(0.76)$ & $-0.04[-0.13,0.06] ; 0.442^{c}$ \\
\hline $\mathrm{CHV}$ visited home within 48 hours of birth, $\mathrm{n}(\%)$ & $105(13 \%)$ & $97(14 \%)$ & $0.98[0.68,1.43] ; 0.92$ \\
\hline Child received OPV 0 vaccine, $n(\%)^{b}$ & $474(83 \%)$ & $468(89 \%)$ & $0.98[0.93,1.03] ; 0.356$ \\
\hline Median days from birth to child receiving OPV 0 vaccine ${ }^{b}$ & 13 & 7 & $8.02[3.06,12.98] ; 0.002^{c}$ \\
\hline Child alive at time of interview, n (\%) & $772(98 \%)$ & $675(98 \%)$ & $1.00[0.98,1.02] ; 0.942$ \\
\hline
\end{tabular}


delayed child immunization for their first oral polio vaccine. Several recent studies have explored the impact of strikes by health workers on health services and outcomes in Kenya [4-9]. These studies mostly rely on hospital and inpatient data, and our study contributes important community-level data on outpatient and antenatal care to the literature. A study using hospital admissions data at 13 County referral hospitals during the 2016-2017 nationwide physicians' and nurses' strikes found that inpatient admissions decreased significantly during strike months, with the authors estimating that 183,170 individuals were expected but did not receive inpatient care at these hospitals over the course of the strikes, including 60,000 maternity patients [4]. Another study using records from 18 County referral hospitals and 14 faith-based health facilities found that the proportion of fully immunized infants fell $57 \%$ in public facilities and increased $252 \%$ in faith-based ones during the 2017 nurses' strike [9]. In our study, a lower proportion of children received their first oral polio vaccine in the strike group ( $83 \%$ versus $89 \%$ ) but this was not statistically significant, although children in the strike group did receive their vaccine significantly later after birth.

While our study was not designed to detect differences in maternal or child mortality, studies reported mixed findings on the association between strikes by health workers and mortality in Kenya [6, 7]. In the only study to date to use population-level health outcomes data, during six strikes by health workers ranging from 9 to 42 days between 2010 and 2016 in Kilifi County, Kenya there was no change in all-cause or cause-specific mortality during strike versus non-strike days [8]. The authors suggest a number of potential explanations for their findings including service delivery continuing in private and faith-based facilities, relatively short duration of strikes, decreased exposure to poor quality inpatient care and high-risk procedures, and the fact that a large proportion of deaths (during strike and nonstrike periods) occur outside of health facilities. Population-level data on maternal and child health utilization and outcomes during the study period were not publicly available at the time of writing, but these data will provide important additional insights into the impact of strikes in 2017.

Given that the strikes in 2017 lasted longer than previous strikes in Kenya, it is possible that they were more likely to affect population-level mortality that is not captured by studies using inpatient data [29, 30]. Decreased ANC coverage during strikes could have also interrupted prevention of mother-to-child transmission services for women living with HIV, but there are no published studies investigating strike-related changes in mother-tochild transmission of HIV. However, even in the control group, our study found that only $70 \%$ of participants attend 4 or more ANC visits and only $73 \%$ delivered at a health facility with a skilled health worker when there were no health worker strikes. Several studies have suggested that the poor quality of facility-based maternal and child health care may account for slower reductions in maternal and neonatal mortality compared to increases in service utilization and facility-based care [11, 31-33]. Research also shows that perceptions of poor quality maternal and child health services among mothers, including long wait times, lack of providers and essential equipment and drugs, disrespectful care, and out-of-pocket payments, represent important barriers to seeking care [34].

It is important to note that many of issues related to poor quality of care and ill-equipped providers and facilities have been at the center of labor disputes and recent strikes in Kenya, with health workers' unions demanding increased investment in the public health sector. It is unclear whether recent strikes have led to improvements in health care services for patients and improved working conditions for providers but addressing the underlying issues of strikes will be critical to efforts for health systems strengthening and universal health coverage, including maternal and child health care. Since this study, subsequent strikes by frontline health workers in Kenya as well as in countries around the world during the COVID-19 pandemic provide further evidence that strikes represent an important but understudied phenomenon in health systems and services research [35].

There are several limitations to this study. First, we relied on retrospective data from Mother-Baby Booklets and self-report to measure maternal and child utilization of services and outcomes. Significant missing data on early child immunizations meant that only data on the oral polio vaccine was included in the analysis, and we could not examine the impact of strikes on other immunizations across the first years of life. In addition to missing data, there was also the potential for recall bias for women who self-reported outcomes. We did not find evidence of increased pregnancy complications or child mortality, however, $37 \%$ of women from the parent study strike group were not able to be re-consented. The only demographic data available on missing women was age, which did not differ between missing and reenrolled women. We were also not able to measure maternal mortality and it is possible that some women who were not able to be relocated had in fact died or had other complications.

Second, we present data from only one County in Kenya. While our findings are generally consistent with the literature available from other parts of Kenya, it is possible that maternal and child health services and 
outcomes were affected differently by strikes in other parts of Kenya. Trans Nzoia is poorer, more rural, and has generally worse maternal and child health indicators by comparison to Kenya overall, however, it does have a mostly representative split between public and private health facilities [22]. Third, we did not assess the impact of a specific health worker strike. Most participants in the strike group delivered during a nationwide nurses' strike but were also pregnant at some point during the physicians' strike earlier in 2017. Moreover, it is common in health facilities when one cadre of health worker is on strike, services may be suspended, and other cadres of health workers may not be working. Thus, it is difficult to assess exactly what health facilities and services remained functional at different times in 2017.

Finally, there were changes in the health system outside of the strike that may have affected maternal and child health services and utilization from 2017 to 2018 and utilization rates of health services tend to increase over time [36]. Thus, it is possible that some of the changes we observed were the result of temporal trends and policy changes. There are several reasons that we believe that temporal trends and policy changes do not wholly account for the significant differences in ANC and health facility deliveries we report between our strike and control study cohorts. As noted previously, in our study population only $15 \%$ of women had health insurance at the time of delivery in 2017 , but $59 \%$ had health insurance at the time of delivery in 2018. This dramatic increase was likely due to the roll out of the Linda Mama insurance program that allows women to access maternity services for free in accredited public and private sector facilities, which was launched in 2017 but due to strikes and other implementation challenges most women were not able to enroll into the program until the following year [15]. To account for the potential impact of Linda Mama insurance enrollment on outcomes of interest, we adjusted for enrollment in health insurance as well as socioeconomic status in our analyses. Moreover, it is not clear that enrollment in Linda Mama has necessarily increased access to and utilization of services. A recent interrupted time-series analysis of ANC and facility deliveries in Kenya found that, controlling for periods of strikes by health workers, there were no significant changes in ANC or facility deliveries in public or private facilities between 2017 (when Linda Mama was launched) up to May 2019 [37]. Taken together with a study using two-year data pooled from 13 County public hospitals between January 2016 and December 2017 that shows a sharp decline and then rebound in admissions to maternity wards [4], our study provides further support that recent health workers' strikes are associated with lower utilization of maternal and child health services.

\section{Conclusion}

We found that access to and utilization of basic maternal and child health services were negatively impacted for pregnant women in Trans Nzoia during nationwide strikes by health workers in 2017. Our study findings are consistent with studies using inpatient and outpatient admissions data in Kenya and provide important community-level data on maternal and child health utilization. Strategies to maintain WHO-recommended maternal and child health services during strikes, particularly for those communities that rely on public sector services, are urgently needed.

\section{Acknowledgements}

The study team would like to thank the study participants for volunteering their time and sharing deeply personal information with the study team. We would also like to thank the community health volunteers who helped us identify participants for study enrollment.

\section{Authors' contributions}

MLS, LYM, JS, LR, and ACD were responsible for the initial study concept and design. JEI, AJ, GA, SC, JCK led the collection of study data. MLS and JNB led the analysis of study data. MLS led the drafting of the initial manuscript. All authors provided critical feedback on various drafts of the manuscript. All authors approved the final version of the manuscript. JS, LR, and ACD served as co-principal investigators for this study as well as the parent study and are recognized by the authorship team as co-senior authors on the manuscript.

\section{Funding}

The lead author (MLS) was supported by a Fulbright Student Research Award from the US Department of State's Bureau of Educational and Cultural Affairs and a Fogarty Global Health Fellowship through the Northern Pacific Global Health Research Fellows Training Program (Grant number D43TW009345). This study was also supported by a grant from Grand Challenges Canada (Grant number 0755-03) to ACD, LR, JS. The funding agencies had no role in the design of the study, the collection, analysis, and interpretation of data, or in the writing of the manuscript.

\section{Availability of data and materials}

The dataset generated during the current study are not publicly available. The study dataset is available from the corresponding author on reasonable request.

\section{Declarations}

Ethics approval and consent to participate

All study participants provided written informed consent to participate in study activities. Participants did not receive any compensation for participation. Participants were assigned a random study ID which was used to identify them in analysis. The study was approved by the Moi University and Moi Teaching and Referral Hospital Institutional Research and Ethics Committee (protocol: IREC/2016/269) in Eldoret, Kenya and the Indiana University School of Medicine Institutional Review Board (protocol: 34173354) in Indianapolis, Indiana, USA. All methods were performed in accordance with the relevant guidelines and regulations.

Consent for publication

Not applicable.

Competing interests

The authors declare that they have no competing interests. 


\section{Author details}

'Indiana University Center for Global Health, 702 Rotary Circle, Suite RO 101, Indianapolis, Indiana, USA. ${ }^{2}$ Academic Model Providing Access to Healthcare (AMPATH), Eldoret, Kenya. ${ }^{3}$ Department of Medicine and Pediatrics, Massachusetts General Hospital, Boston, MA, USA. ${ }^{4}$ Department of Obstetrics and Gynaecology, University of British Columbia, Vancouver, Canada. ${ }^{5}$ Department of Medicine, Indiana University School of Medicine, Indianapolis, IN, USA. ${ }^{6}$ Department of Child Health and Paediatrics, College of Health Sciences, School of Medicine, Moi University, Eldoret, Kenya. ${ }^{7}$ Department of Obstetrics and Gynaecology, University of Toronto, Toronto, Canada.

Received: 2 May 2021 Accepted: 19 August 2021 Published online: 01 September 2021

\section{References}

1. Salama P, Mclsaac M, Campbell J. Health workers' strikes: a plea for multisectoral action. Bull World Health Organ. 2019;97(7):443-A.

2. McCollum R, Limato R, Otiso L, Theobald S, Taegtmeyer M. Health system governance following devolution: comparing experiences of decentralisation in Kenya and Indonesia. BMJ Glob Health. 2018;3(5): e000939. https://doi.org/10.1136/bmjgh-2018-000939.

3. Mbindyo $P$, Blaauw $D$, English $M$. The role of clinical officers in the Kenyan health system: a question of perspective. Hum Resour Health. 2013;11(1):32. https://doi.org/10.1186/1478-4491-11-32.

4. Irimu G, Ogero M, Mbevi G, Kariuki C, Gathara D, Akech S, et al. Tackling health professionals' strikes: an essential part of health system strengthening in Kenya. BMJ Glob Health. 2018;3(6):e001136. https://doi. org/10.1136/bmjgh-2018-001136

5. Njuguna J. Impact of health Workers' strike in august 2014 on health Services in Mombasa County Referral Hospital, Kenya. J Health Care Poo Underserved. 2015;26(4):1200-6. https://doi.org/10.1353/hpu.2015.0106.

6. Adam MB, Muma S, Modi JA, Steere M, Cook N, Ellis W, et al. Paediatric and obstetric outcomes at a faith-based hospital during the 100-day public sector physician strike in Kenya. BMJ Glob Health. 2018;3(2):e000665. https:// doi.org/10.1136/bmjgh-2017-000665.

7. Kaguthi GK, Nduba V, Adam MB. The impact of the nurses', doctors' and clinical officer strikes on mortality in four health facilities in Kenya. BMC Health Serv Res. 2020;20(1):469. https://doi.org/10.1186/s12913-020-05337-9.

8. Ong'ayo G, Ooko M, Wang'ondu R, Bottomley C, Nyaguara A, Tsofa BK, et al. Effect of strikes by health workers on mortality between 2010 and 2016 in Kilifi, Kenya: a population-based cohort analysis. Lancet Glob Health. 2019; 7(7):e961-e7. https://doi.org/10.1016/S2214-109X(19)30188-3.

9. Njuguna J. Impact of Nurses' strike in Kenya on number of fully immunized infants in 18 county referral hospitals. J Health Care Poor Underserved. 2018; 29(4):1281-7. https://doi.org/10.1353/hpu.2018.0095.

10. Keats EC, Ngugi A, Macharia W, Akseer N, Khaemba EN, Bhatti Z, et al. Progress and priorities for reproductive, maternal, newborn, and child health in Kenya: a countdown to 2015 country case study. Lancet Glob Health. 2017;5(8):e782-e95. https://doi.org/10.1016/S2214-109X(17)30246-2.

11. Owolabi O, Riley T, Juma K, Mutua M, Pleasure ZH, Amo-Adjei J, et al. Incidence of maternal near-miss in Kenya in 2018: findings from a nationally representative cross-sectional study in 54 referral hospitals. Sci Rep. 2020; 10(1):15181. https://doi.org/10.1038/s41598-020-72144-x.

12. WHO U, UNFPA, World Bank Group, and the UN Population Division. Trends in maternal mortality: 2000 to 2017. Geneva: World Health Organization [Available from: https://data.worldbank.org/indicator/SH.STA.MMRT?loca tions=KE]; 2019

13. Kenya Demographic and Health Survey, 2014. Nairobi: Kenya National Bureau of statistics.

14. Masaba BB, Mmusi-Phetoe RM. Free maternal health care policy in Kenya: level of utilization and barriers. Int J Africa Nurs Sci. 2020;13:100234. https:// doi.org/10.1016/j.jijans.2020.100234.

15. Linda Mama services. Nairobi: National Hospital Insurance Fund, Kenya [Available from: http://www.nhif.or.ke/healthinsurance/lindamamaServices].

16. Dennis ML, Benova L, Abuya T, Quartagno M, Bellows B, Campbell OMR. Initiation and continuity of maternal healthcare: examining the role of vouchers and user-fee removal on maternal health service use in Kenya. Health Policy Plan. 2019;34(2):120-31. https://doi.org/10.1093/heapol/czz004.
17. Owuor $\mathrm{H}$, Amolo AS. Interrupted time series analysis of free maternity services policy in Nyamira County. Western Kenya PLoS One. 2019;14(5): e0216158. https://doi.org/10.1371/journal.pone.0216158.

18. Tama E, Molyneux S, Waweru E, Tsofa B, Chuma J, Barasa E. Examining the implementation of the free maternity services policy in Kenya: a mixed methods process evaluation. Int J Health Policy Manag. 2018;7(7):603-13. https://doi.org/10.15171/ijhpm.2017.135.

19. Kabia E, Mbau R, Oyando R, Oduor C, Bigogo G, Khagayi S, et al. "We are called the et cetera": experiences of the poor with health financing reforms that target them in Kenya. Int J Equity Health. 2019;18(1):98.

20. Lang'at $E$, Mwanri $L$, Temmerman M. Effects of implementing free maternity service policy in Kenya: an interrupted time series analysis. BMC Health Serv Res. 2019;19(1):645. https://doi.org/10.1186/s12913-019-4462-x.

21. Trans Nzoia County: Health at a Glance Nairobi: Ministry of Health, Government of Kenya and Health Policy Project/USAID; May 2015.

22. Kenya Master Health Facility List: Ministry of Health, Government of Kenya; 2019 [Available from: http://kmhfl.health.go.ke/\#/home].

23. Bwayo P. Nurses in Trans Nzoia County call of their 44-day strike. Daily Nation (Kenya). 2017 March 24, 2017.

24. Maldonado LY, Bone J, Scanlon ML, Anusu G, Chelagat S, Jumah A, et al Improving maternal, newborn and child health outcomes through a community-based women's health education program: a cluster randomised controlled trial in western Kenya. BMJ Glob Health. 2020;5:12.

25. Scanlon ML, Maldonado LY, Ikemeri JE, Jumah A, Anusu G, Chelagat S, et al 'It was hell in the community': a qualitative study of maternal and child health care during health care worker strikes in Kenya. Preprint Research Square (posted June 3, 2021).https://doi.org/10.21203/rs.3.rs-581857/v1.

26. Harris PA, Taylor R, Minor BL, Elliott V, Fernandez M, O'Neal L, et al. The REDCap consortium: Building an international community of software platform partners. J Biomed Inform. 2019;95:103208.

27. Harris PA, Taylor R, Thielke R, Payne J, Gonzalez N, Conde JG. Research electronic data capture (REDCap)--a metadata-driven methodology and workflow process for providing translational research informatics support. J Biomed Inform. 2009;42(2):377-81. https://doi.org/10.1016/j.jbi.2008.08.010.

28. Zou GY, Donner A. Extension of the modified Poisson regression model to prospective studies with correlated binary data. Stat Methods Med Res. 2013;22(6):661-70. https://doi.org/10.1177/0962280211427759.

29. The L. Kenya's nurses strike takes its toll on health-care system. Lancet. 2017; 389(10087):2350.

30. Williams PCM. The reality of the mortality statistics of the nurses' strike in Kenya. Lancet. 2017;390(10094):551. https://doi.org/10.1016/S0140-6736(1 7)31944-X.

31. Chou VB, Walker N, Kanyangarara M. Estimating the global impact of poor quality of care on maternal and neonatal outcomes in 81 low- and middleincome countries: a modeling study. PLoS Med. 2019;16(12):e1002990. https://doi.org/10.1371/journal.pmed.1002990.

32. Fink G, Ross R, Hill K. Institutional deliveries weakly associated with improved neonatal survival in developing countries: evidence from 192 Demographic and health surveys. Int J Epidemiol. 2015;44(6):1879-88. https://doi.org/10.1093/ije/dyv115.

33. Gage AD, Carnes F, Blossom J, Aluvaala J, Amatya A, Mahat K, et al. In lowand middle-income countries, is delivery in high-quality obstetric facilities geographically feasible? Health Aff (Millwood). 2019;38(9):1576-84. https:// doi.org/10.1377/hlthaff.2018.05397.

34. Ansu-Mensah M, Danquah Fl, Bawontuo V, Ansu-Mensah P, Kuupiel D. Maternal perceptions of the quality of Care in the Free Maternal Care Policy in sub-Sahara Africa: a systematic scoping review. BMC Health Serv Res. 2020;20(1):911. https://doi.org/10.1186/s12913-020-05755-9.

35. Essex R, Weldon SM. Health care worker strikes and the Covid pandemic. N Engl J Med. 2021;384(24):e93. https://doi.org/10.1056/NEJMp2103327.

36. Doctor HV, Radovich E, Benova L. Time trends in facility-based and privatesector childbirth care: analysis of Demographic and health surveys from 25 sub-Saharan African countries from 2000 to 2016. J Glob Health. 2019;9(2): 020406. https://doi.org/10.7189/jogh.09.020406.

37. Orangi S, Kairu A, Malla L, Ondera J, Mbuthia B, Ravishankar N, et al. Impact of free maternity policies in Kenya: an interrupted time-series analysis. BMJ Glob Health. 2021;6(6).

\section{Publisher's Note}

Springer Nature remains neutral with regard to jurisdictional claims in published maps and institutional affiliations. 